\title{
Automatic Recognition of Ventricular Abnormal Potentials in Intracardiac Electrograms
}

\author{
Giulia Baldazzi ${ }^{1,2}$, Marco Orrù ${ }^{1}$, Mirko Matraxia $^{3}$, Graziana Viola ${ }^{4}$, Danilo Pani ${ }^{1}$ \\ ${ }^{1}$ Department of Electrical and Electronic Engineering, University of Cagliari, Cagliari, Italy \\ ${ }^{2}$ DIBRIS, University of Genova, Genova, Italy \\ ${ }^{3}$ Medical Concept Lab, Sassari, Italy \\ ${ }^{4}$ Division of Cardiology, San Francesco Hospital, Nuoro, Italy
}

\begin{abstract}
Ventricular abnormal potentials are low-amplitude electrical signals that appear in intracardiac electrograms during a QRS or with an unpredictable delay with respect to it. Their spatial localization can be exploited by cardiologists for the identification of the ablation targets in substrate-guided mapping and ablation procedures. In this work, an automatic approach for a reliable detection of such potentials in intracardiac electrograms is proposed.

To this aim, 86 intracardiac electrograms from five patients with post-ischemic ventricular tachycardia, acquired by the CARTO3 System, were retrospectively annotated by an expert cardiologist, to be used for a supervised classifier training and test.

The automatic detection was based on a non-linear denoising followed by a time-scale decomposition based on the continuous wavelet transform. Then, different morphological features were extracted from both the time-scale domain and the time domain, and used to feed a support vector machine trained to discriminate between physiological and abnormal potentials. The recognition accuracy exceeded 93\%, paving the way to further developments and more extensive studies.
\end{abstract}

\section{Introduction}

Ventricular tachycardia (VT) is a potentially lethal form of ventricular arrhythmia, that could descend from several ischemic and non-ischemic causes. One of the elective tools for the treatment is substrate-guided mapping and catheter ablation during sinus rhythm [1], [2]. This procedure allows ablating multiple VT morphologies and is effective in the prevention of arrhythmia recurrences in scar-related unmappable VT [3]. Substrate-guided mapping requires an invasive pointby-point acquisition of endocardial potentials through intracardiac catheters. Abnormal voltage cut-off values for bipolar electrograms are established: $\mathrm{V}<0.5 \mathrm{mV}$ for dense scar, $0.5<\mathrm{V}<1.5 \mathrm{mV}$ for abnormal tissue [4].

The main purpose of substrate ablation is targeting and eliminating areas of slow conduction associated to abnormal potentials (APs), within the damaged substrate, defined during sinus rhythm (SR) [1]-[3]. Several definitions of AP exist: late potential or early potential, according to its location within or outside the surface QRS support [5], or a fractionated, late, isolated potential or local abnormal activity [6].

The identification of AP is an operator-dependent process supported by the use of multielectrode catheters and different tools integrated in off-the-shelf 3D mapping systems such as Rhythmia HDx (Boston Scientific, Cambridge, MA, USA), CARTO3v6, (Biosense Webster, Diamond Barr, CA, USA), EnSite Precision (Abbott, Chicago, IL, USA) [7], [8]. Tools such as CARTO3 Ripple Mapping Module, which provides a dynamic visual representation of bipolar electrograms shown as bars [9], are time-domain based. A frequency-domain index [10], based only on the Hilbert-Huang transform, was developed to recognize abnormal high-frequency potentials during $\mathrm{SR}$ and to identify ventricular tachycardia isthmuses.

In this work, in order to obtain a shorter ablation procedure and to optimize the outcome, an automatic tool for detection and classification of AP is presented and evaluated on real recordings from five subjects affected by post-ischemic VT. Time-scale features obtained after continuous wavelet transform (CWT) have been associated to time-domain features to train a supervised classifier to accomplish the classification of AP. This choice is motivated by different time and frequency 
domain features characterizing APs compared to physiological potentials.

\section{Background}

Wavelet transform has been widely adopted in biomedical signal processing, especially with the aim of dealing with transient and non-stationary signals [11]. It relies on a time-scale representation of the signal $f(t)$ through a set of coefficients, that in case of the CWT are obtained as:

$$
W T_{f}(a, b)=\frac{1}{\sqrt{a}} \int_{R} \psi^{*}\left(\frac{t-b}{a}\right) f(t) d t
$$

where $a \in \mathrm{R}^{+}$and $b \in \mathrm{R}$ represent the scale and timeshift parameters, respectively, whereas $\frac{1}{\sqrt{a}} \psi\left(\frac{t-b}{a}\right)$ represents a time-shifted and scaled version of a specific function $\psi$, called mother wavelet. In other words, for every translation in time, each wavelet coefficient represents a similarity index between of the signal and the scaled version of $\psi$. Specifically, the scale factor regulates the degree of stretching of the mother wavelet and, as such, is inversely linked to the frequency: small scales generate a contracted $\psi$, so higher signal frequency components are examined; conversely, larger scales are associated to a more dilated $\psi$ and so to lower frequency contributions. This approach leads to a variable support of the basis functions in close relationship with the explored frequency band, which is not possible with the Fourier analysis. As such, unlike Fourier analysis, wavelet transform guarantees a good resolution both in time and in frequency domain, which can be exploited to identify APs. While in the discrete wavelet transform the scale parameter is varied discretely by powers of two, in the CWT there is not such constraint. However, in the computer implementation of the technique, the scale parameter is also discretized but finely, e.g. as fractional powers of two.

\section{Materials and methods}

At first, each electrogram underwent a wavelet denoising stage. A translation-invariant $\grave{a}$ trous (undecimated) algorithm was adopted, to achieve translation-invariance at a low computational cost compared to other approaches [12].

We choose the Daubechies2 mother wavelet and a decomposition level equal to 2 , in order to implement the denoising only on the upper part of the signal band, i.e. from the folding frequency down to about $125 \mathrm{~Hz}$. In this way, we tried to remove under-threshold small fluctuations of the signal that could represent confounding factors for the subsequent analysis. The
Universal threshold (2) and the soft-thresholding approach of the details (3) were also preferred at this stage:

$$
\begin{gathered}
\theta_{j}=\sigma_{j} \cdot \sqrt{2 \ln (N)} \\
\overline{c D_{j, k}}=\left\{\begin{array}{cl}
\operatorname{sign}\left(c D_{j, k}\right) \cdot\left(\left|c D_{j, k}\right|-\theta_{j}\right) & \text { if }\left|c D_{j, k}\right| \geq \theta_{j} \\
0 & \text { otherwise }
\end{array}\right.
\end{gathered}
$$

where $c D_{j, k}$ indicates the $k$-th detail coefficients at level $j$, while $\sigma_{j}$ was calculated as:

$$
\sigma_{j}=1.4826 \cdot \operatorname{median}\left(\left|c D_{j}\right|\right)
$$

On the denoised electrogram, a window of $50 \mathrm{~ms}$ before and $300 \mathrm{~ms}$ after the reference point was identified as the portion of interest for the analysis. Each reference point was selected during the clinical procedure and saved automatically by the CARTO3 system.

\subsection{Feature extraction and classification}

The CWT decomposition of the denoised windowed signal was computed by exploiting the Daubechies2 mother wavelet. All the scales from 2 to 35 were considered, in order to analyze the spectral components of the signal between about $16 \mathrm{~Hz}$ and $500 \mathrm{~Hz}$. Then, the average power on each scale $a$ was evaluated as:

$$
\check{P}(a)=\frac{1}{N} \sum_{i=1}^{N} W T_{f}(a, i)^{2}
$$

where $N$ is the total number of wavelet coefficients at that scale.

For each bipolar electrogram, the five scales with the highest $\breve{P}(a)$ were selected. Then, the standard deviation of such $\breve{P}(a)$ and their sum were computed and considered as features in the time-scale domain. Moreover, two time-domain features were extracted. The first one is the peak-to-peak amplitude of the bipolar electrogram. This measure was meant to help distinguishing between high voltage signals $(>1.5 \mathrm{mV})$, which are typically associated to normal myocardial areas, from those identified as border zones or scars [4].

Then, since pathological electrograms are generally characterized by highly fragmented trends [13], we also considered the total number of peaks in the windowed signal. To this aim, we computed the absolute value of the window and counted all peaks exceeding $75 \%$ of its mean absolute deviation value.

Finally, all these features were used to train a support vector machine (SVM) with Gaussian radial basis function (RBF) kernel, $v=0.5$, and box constraint set to 1. Since the dataset is quite small, in order to avoid overfitting, default parameters were used.

In order to train the classifier, a retrospective analysis was performed on a small dataset composed of 86 signal windows acquired in SR from five patients with post- 
ischemic VT, during electroanatomic mapping. Recordings were performed at the San Francesco Hospital (Nuoro, Italy) from 2017 to 2018. During the procedure, radiofrequency ablation followed the usual clinical protocols.

Bipolar intracardiac electrograms from such routine procedures were acquired by the CARTO 3 system at a sampling frequency of $1 \mathrm{kHz}$ and band-pass filtered between 16 and $500 \mathrm{~Hz}$ by the acquisition system itself. The duration of each signal is $2.5 \mathrm{~s}$, but only the last beat guaranteed to be related to an effective contact between the catheter and the myocardium. The signals were manually annotated by an expert cardiologist, for physiological potentials and APs (including both late and early potentials [5]), by exploiting the intracardiac signals and the corresponding simultaneous surface ECG recordings.

\subsection{Performance evaluation}

To evaluate the performance of the classifier, four indices were estimated: the accuracy (ACC), the True Positive Rate (TPR) or Sensitivity, the True Negative Rate (TNR) or Specificity and the F1-score:

$$
\begin{gathered}
\mathrm{ACC}=\frac{\mathrm{TP}+\mathrm{TN}}{\mathrm{P}+\mathrm{N}} \times 100 \\
\mathrm{TPR}=\frac{\mathrm{TP}}{\mathrm{P}} \times 100 \\
\mathrm{TNR}=\frac{\mathrm{TN}}{\mathrm{N}} \times 100 \\
\mathrm{~F} 1_{\text {score }}=2 \frac{\text { Precision } \cdot \mathrm{TPR}}{\text { Precision }+\mathrm{TPR}}
\end{gathered}
$$

where Positive $(\mathrm{P})$ and Negative $(\mathrm{N})$ are respectively the pathologic and physiologic target classes, True Positive (TP) and True Negative (TN) are respectively the number of pathologic and physiologic observations correctly classified, while the precision is the ratio between TP and the number of observations predicted as pathological. Moreover, the receiver operating characteristic (ROC) and the area under the curve (AUC) were also adopted to quantify the classifier performance.

SVM classifier was trained and tested ten times, each time by using a 10 -fold cross-validation scheme. In this way, the mean and the standard deviation from the ten average values (across the 10 folds) of each performance index were computed.

All computations and analyses were performed with Matlab v2018b (MathWorks Inc., MA, USA).

\section{4. $\quad$ Results}

The results of the 10-times 10-fold validation trials are shown in Table 1. The mean and standard deviation of each performance index are reported.

From the analysis of these results, it is easy to recognize that the chosen classifier, exploiting the proposed features, presents optimal results reaching a high value of accuracy, equal to $93.95 \%$ on average. The robustness is confirmed by the very low standard deviation values. The percentages of TPR and TNR are also good and balanced, which means that the classifier managed to recognize the majority of the observations correctly.

F1-score, which by definition assumes the value 1 for high accuracy of the test and 0 for low accuracy of the test, also marks high values.

Table 1. Mean \pm standard deviation of the performance indexes derived from the 10-times 10 fold cross-validation by using the SVM classifier.

\begin{tabular}{l|c} 
Performance index & Mean \pm std \\
\hline ACC [\%] & $93.95 \pm 0.01$ \\
TPR [\%] & $94.00 \pm 0.00$ \\
TNR [\%] & $93.89 \pm 0.01$ \\
F1-score & $0.948 \pm 0.00$
\end{tabular}

All these results are confirmed by the ROC curves and the AUC, shown in Figure 1, each one related to one of the ten trials. Moreover, the AUC values are high in every case, achieving a mean value of 0.97 out of 1 .

Finally, the confusion matrix related to the worst case, shown in Figure 2, reveals that $94 \%$ of AP are correctly classified, giving a false negative rate of $6 \%$ whereas, considering the class of the physiologic potentials, the true negative rate reaches $91.7 \%$.

\section{Conclusions}

In this work, a novel tool for automatic identification of APs in intracardiac electrograms, based on a supervised classifier trained on time-scale and time-domain features, was conceived and evaluated. To this aim, real recordings from five subjects with post-ischemic VT acquired during routine procedures were used. Classification results showed a very high accuracy of the proposed approach, with low false rates for both pathological and physiological classes. Even though the results are encouraging, their generalizability should be carefully considered because of the limited size of the dataset. Moreover, signal annotation was performed by a single expert cardiologist, which could have led to a biased response. Deeper analyses on larger datasets could 


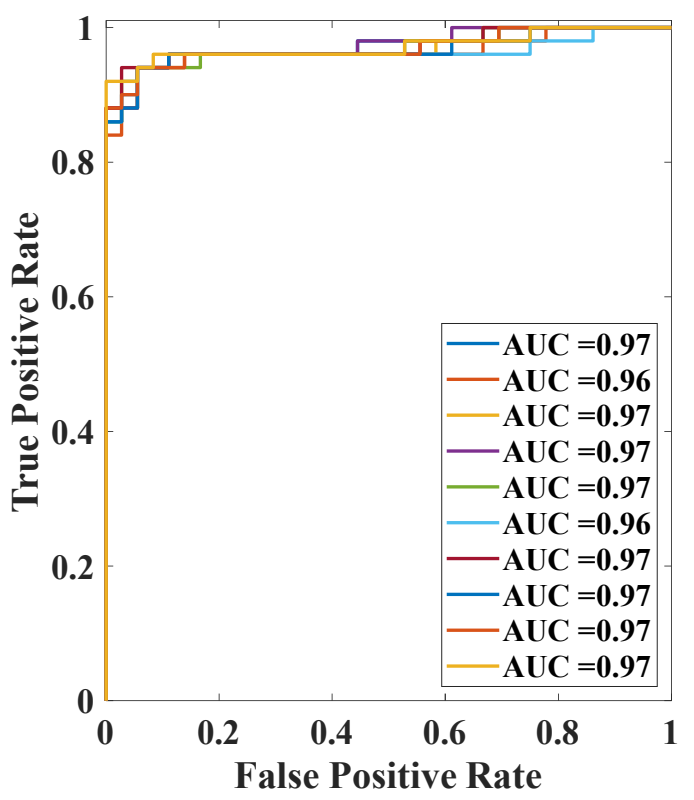

Figure 1. ROC curves and AUC values obtained from the 10-times 10-fold cross-validation.

overcome these limitations and assess the actual exploitability of the proposed approach in clinical procedures.

\section{References}

[1] P. Vergara et al., "Late potentials abolition as an additional technique for reduction of arrhythmia recurrence in scar related ventricular tachycardia ablation," J. Cardiovasc. Electrophysiol., vol. 23, no. 6, pp. 621-627, Jun. 2012.

[2] P. Jaïs et al., "Elimination of local abnormal ventricular activities: a new end point for substrate modification in patients with scar-related ventricular tachycardia," Circulation, vol. 125, no. 18, pp. 2184-2196, 2012.

[3] S. Kumar et al., "Substrate-based ablation versus ablation guided by activation and entrainment mapping for ventricular tachycardia: a systematic review and metaanalysis," J. Cardiovasc. Electrophysiol., vol. 27, no. 12, pp. 1437-1447, Dec. 2016.

[4] M. F. E., C. D. J., G. C. D., and Z. Erica, "Linear ablation lesions for control of unmappable ventricular tachycardia in patients with ischemic and nonischemic cardiomyopathy," Circulation, vol. 101, no. 11, pp. 1288-1296, Mar. 2000.

[5] J. Silberbauer et al., "Noninducibility and late potential abolition: a novel combined prognostic procedural end point for catheter ablation of postinfarction ventricular tachycardia," Circ. Arrhythmia Electrophysiol., vol. 7, no. 3, pp. 424-435, 2014.

[6] S. K. S. Huang, M. A. Wood, and J. M. Miller, Catheter ablation of cardiac arrhythmias E-book. Elsevier Health Sciences, 2010.

[7] F. Bourier et al., "Is it feasible to offer 'targeted ablation' of ventricular tachycardia circuits with better understanding of isthmus anatomy and conduction characteristics?," EP

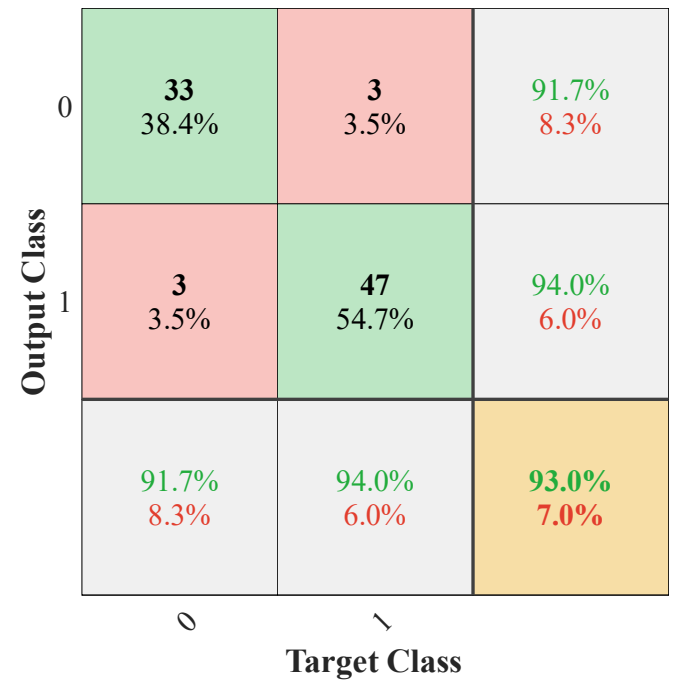

Figure 2. Confusion matrix related to the worst classification case among the ten trials. 0 and 1 represent the physiological and the abnormal population respectively.

Eur., vol. 21, no. Supplement_1, pp. i27-i33, Feb. 2019.

[8] Z. Aziz and R. Tung, "Novel mapping strategies for ventricular tachycardia ablation," Current Treatment Options in Cardiovascular Medicine. 2018.

[9] L. Vishal et al., "A prospective study of ripple mapping the post-infarct ventricular scar to guide substrate ablation for ventricular tachycardia," Circ. Arrhythmia Electrophysiol., vol. 9, no. 6, p. e004072, Jun. 2016.

[10] C.-Y. Lin et al., "Simultaneous amplitude frequency electrogram transformation (SAFE-T) mapping to identify ventricular tachycardia arrhythmogenic potentials in sinus rhythm," JACC Clin. Electrophysiol., vol. 2, no. 4, pp. 459470,2016

[11] L. Sörnmo and P. Laguna, Bioelectrical signal processing in cardiac and neurological applications, vol. 8. Academic Press, 2005.

[12] D. Pani et al., "Real-time neural signals decoding onto offthe-shelf DSP processors for neuroprosthetic applications," IEEE Trans. Neural Syst. Rehabil. Eng., vol. 24, no. 9, 2016.

[13] B. Campos, M. E. Jauregui, F. E. Marchlinski, S. Dixit, and E. P. Gerstenfeld, "Use of a novel fragmentation map to identify the substrate for ventricular tachycardia in postinfarction cardiomyopathy," Hear. Rhythm, vol. 12, no. 1, pp. 95-103, Jan. 2015.

Address for correspondence:

Danilo Pani, Ph.D.

Department of Electrical and Electronic Engineering

University of Cagliari

Cagliari, Italy

danilo.pani@unica.it 